\title{
Effects of different forms of monocular deprivation on primary visual cortex maps
}

\author{
SAJJIDA JAFFER, ${ }^{1, *}$ VASILY VOROBYOV, ${ }^{1,2}$ AND FRANK SENGPIEL ${ }^{1}$ \\ ${ }^{1}$ School of Biosciences, Cardiff University, Cardiff, United Kingdom \\ ${ }^{2}$ Institute of Cell Biophysics, Russian Academy of Sciences, Pushchino, Russia \\ *Present address: School of Biological Sciences, University of Reading, Hopkins Building, Reading, RG6 6UB, UK
}

(Received June 1, 2012; Accepted July 10, 2012; First Published Online August 13, 2012)

\begin{abstract}
Monocular deprivation (MD) by lid suture is one of the classic paradigms for the study of developmental plasticity in the cerebral cortex, and we have detailed knowledge of its anatomical and physiological consequences as well as underlying molecular and cellular mechanisms. However, the effects of other forms of manipulating visual input through one eye on the functional architecture of the primary visual cortex (V1) have not yet been examined directly. We compared MD by lid suture with the effects of daily monocular lens wear using either a frosted lens or a neutral density (ND) filter. We used optical imaging of intrinsic signals and visually evoked potentials (VEPs) to assess responses in V1 to monocular stimulation. We found that loss of stimulus contrast through monocular frosted lens wear resulted in marked takeover of cortical territory by the nondeprived eye (NDE) similar to that caused by classic MD, and in virtual absence of orientation-selective responses following stimulation of the deprived eye (DE). Furthermore, amplitudes of VEPs in response to gratings of a range of spatial frequencies were significantly reduced in the DE compared to the NDE. In contrast, differences in luminance between two eyes caused by an ND filter in front of one eye did not affect ocular dominance and orientation maps, and there was no significant difference in the amplitude of VEPs elicited through the two eyes. Our results are consistent with previous electrophysiological studies in demonstrating that binocular pattern information is necessary to maintain normal functional maps in both eyes, while reduced luminance in one eye has little effect on the overall functional architecture and visual responses in V1.
\end{abstract}

Keywords: Ocular dominance, Orientation selectivity, Contrast, V1, Optical imaging

\section{Introduction}

The primary visual cortex (V1) is one of the model systems for the study of experience-dependent plasticity. The classical experiments by Wiesel and Hubel (1963) were the first to demonstrate that monocular deprivation (MD) produced experimentally by unilateral lid suture during the critical period (Hubel \& Wiesel, 1970) results in the majority of cells being driven exclusively by the nondeprived eye (NDE); deprived eye (DE) afferents shrink (Shatz \& Stryker, 1978; Antonini \& Stryker, 1996) and the animal is effectively rendered blind on that eye (Dews \& Wiesel, 1970). MD by lid suture prevents form vision and it reduces the levels of retinal illumination by 2-5 log units depending on pigmentation, wavelength, and state of adaptation (Wiesel \& Hubel, 1963; Crawford \& Marc, 1976; Loop \& Sherman, 1977); however, it does permit entry of diffuse light, permitting cats to make brightness discriminations (Loop \& Sherman, 1977), and $30 \%$ of the cells respond to visual stimuli presented through the sutured lids (Spear et al., 1978).

In addition to MD by lid suture, several studies have employed other methods of MD, which either distort pattern information or reduce retinal illumination to determine which factor is the principal

Address correspondence and reprint requests to: Frank Sengpiel, School of Biosciences, Cardiff University, Museum Avenue, Cardiff, CF10 3AX, UK. E-mail: SengpielF@cf.ac.uk cause of the observed effects on cortical function and behavioral outcome. Single-cell recordings have shown that attenuating retinal illumination by using neutral density (ND) filters (which have little effect on transmission of contrast information) does not diminish the cortical responses through that eye; however, diffusing or blurring images in one eye with little change in retinal illumination is sufficient to cause ocular dominance (OD) shifts (Blakemore, 1976; Eggers \& Blakemore, 1978; Christen \& Mower, 1987; Rittenhouse et al., 2006). For instance, Blakemore (1976), using an opal perspex disk of an optical density of 0.25 , reported a significant OD shift in one kitten after $37 \mathrm{~h}$ of visual exposure over 4 weeks. Similarly, Rittenhouse et al. (2006) employed a diffusing contact lens and found a significant effect of monocular blur on neuronal OD distributions after $24 \mathrm{~h}$ of exposure over 2 days. Similarly, suturing the nictitating membrane only (which diffuses the retinal image but reduces illumination by just $1-2 \log$ units) produces anatomical and physiological changes as severe as those following eyelid suture (Wiesel \& Hubel, 1963).

It is now clear that the OD shift in cats (Jaffer et al., 2012) like in mice (Frenkel \& Bear, 2004; Mrsic-Flogel et al., 2007) results from two distinct events: depression of DE responses followed by a delayed increase of NDE responses. Our aim was to perform a quantitative comparison of the consequences of monocular blur, attenuated retinal illumination, and lid suture on the functional 
architecture of the cat V1 and on visually evoked potentials (VEPs). Our study was focused on the effects of different forms of MD on the DE responses, which we expected to be near maximal after exposing animals for $4 \mathrm{~h}$ every day for 2 weeks.

\section{Materials and methods}

\section{Rearing details}

All procedures were approved by local ethical review and covered by U.K. Home Office licenses. A total of 12 cats were used in this study. The first group was normally reared and then underwent MD by lid suture under general anesthesia (ketamine, $20-40 \mathrm{mg} / \mathrm{kg}$ i.m., and xylazine, $2-4 \mathrm{mg} / \mathrm{kg}$ i.m.) starting at postnatal day 28 for 7 days $(n=3)$. The second group $(n=3)$ underwent MD with a frosted lens, which virtually eliminated pattern information but reduced retinal illumination by just $50 \%$ (optical density of 0.3 ), and the third group $(n=2)$ wore an ND filter, which left pattern information intact but reduced retinal illumination by $99 \%$ (optical density, 2.0). Modulation transfer functions plotting Michelson contrast $\left(I_{\max }-I_{\min }\right) /\left(I_{\max }+I_{\min }\right)$ against the spatial frequency of sinusoidal test gratings show that the frosted diffuser lens did not transmit any contrast information beyond 0.2 cycles/deg, while the ND filter had little detrimental effect on image contrast (see Fig. 1). An age-matched fifth group was raised in a normal 12-h light/dark cycle and served as a control $(n=4)$. Both the frosted lens and the ND filter lens were fitted within custom-made opaque masks, which were fastened with Velcro bands. For all groups, MD started at postnatal day 28; animals were exposed to a lighted environment for $4 \mathrm{~h}$ daily and kept in a dark room together with their mother the rest of the time. The room in which they were reared contained cardboard boxes, toys, and furniture for environmental enrichment.

A No filter

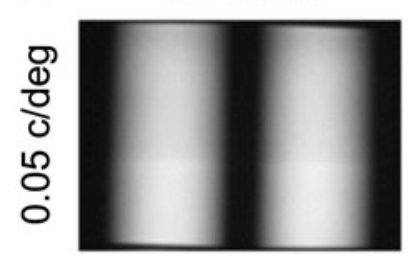
ND filter Frosted lens
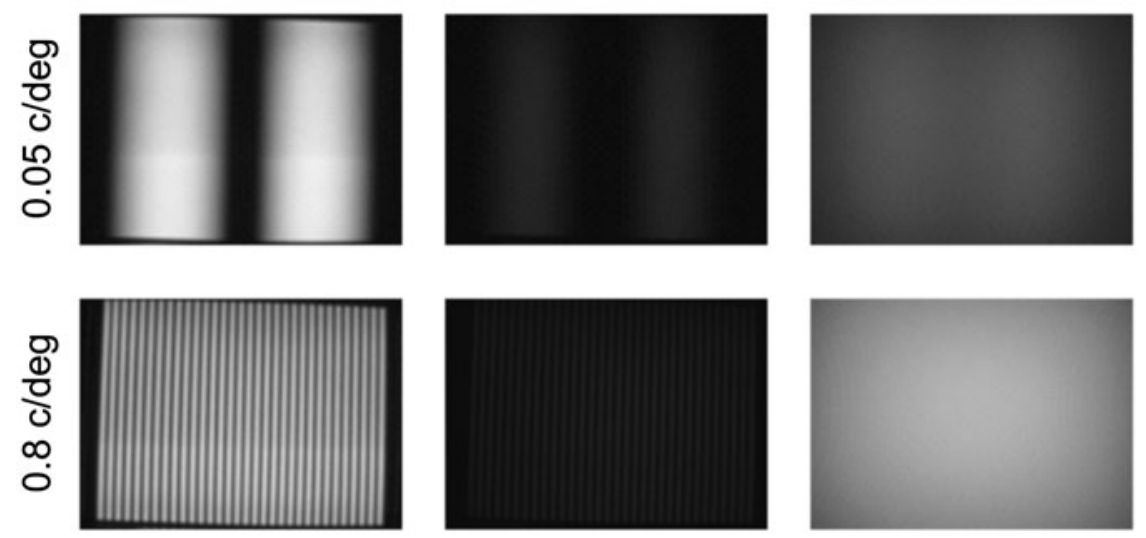

B
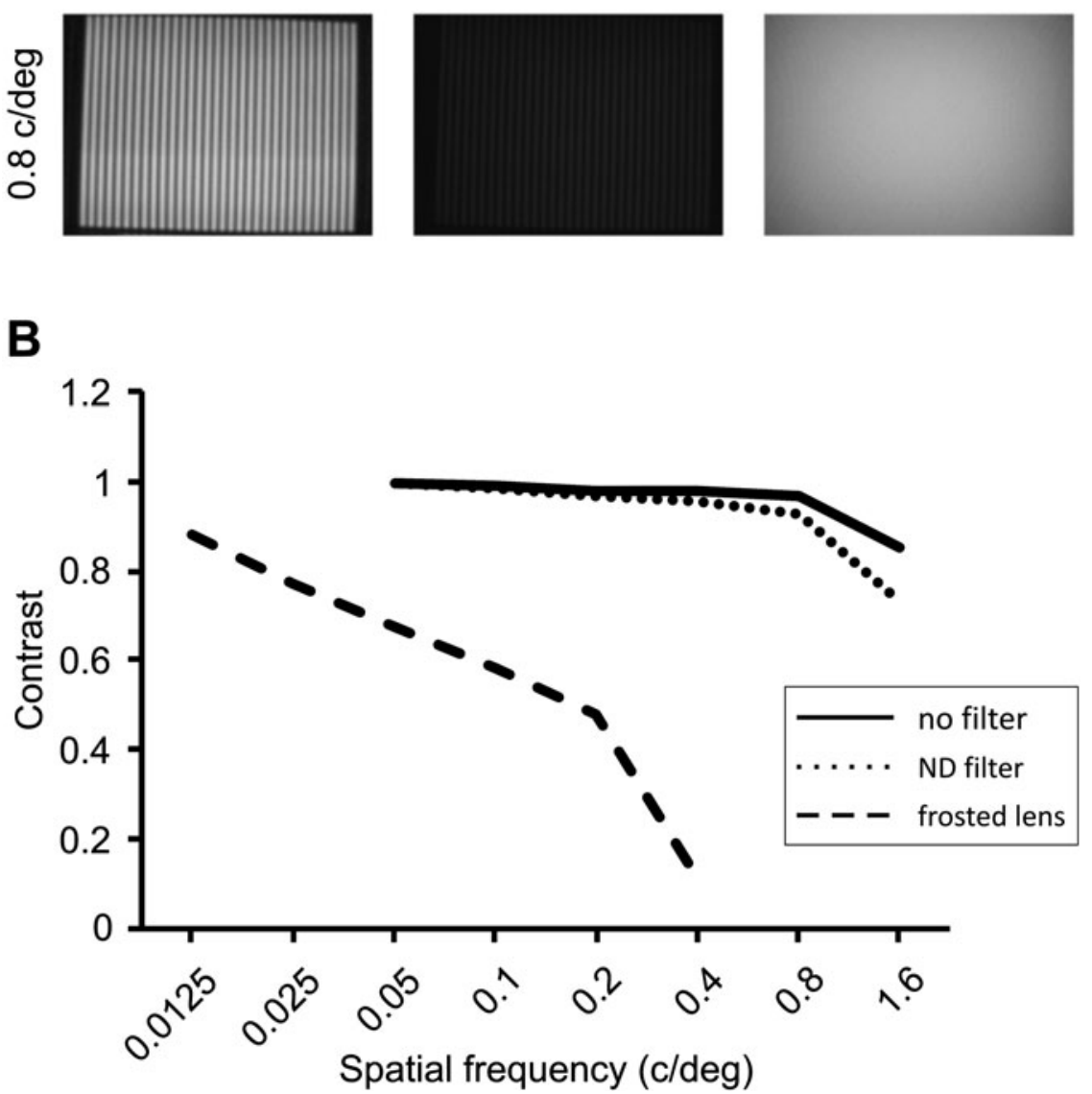

Fig. 1. Transmission of brightness and contrast information through filters used in this study. (A) Appearance of a low spatial frequency grating ( 0.05 cycles/deg; top row) and of a grating of a spatial frequency close to the optimum for cat V1 neurons representing the central visual field (0.8 cycles/deg, bottom row) viewed without filter, through an ND filter of OD2.0 or a frosted diffuser of OD0.3. (B) Modulation transfer functions plotting Michelson contrast against the spatial frequency of sinusoidal test gratings for the two filters compared with the Michelson contrast of the unfiltered gratings. 

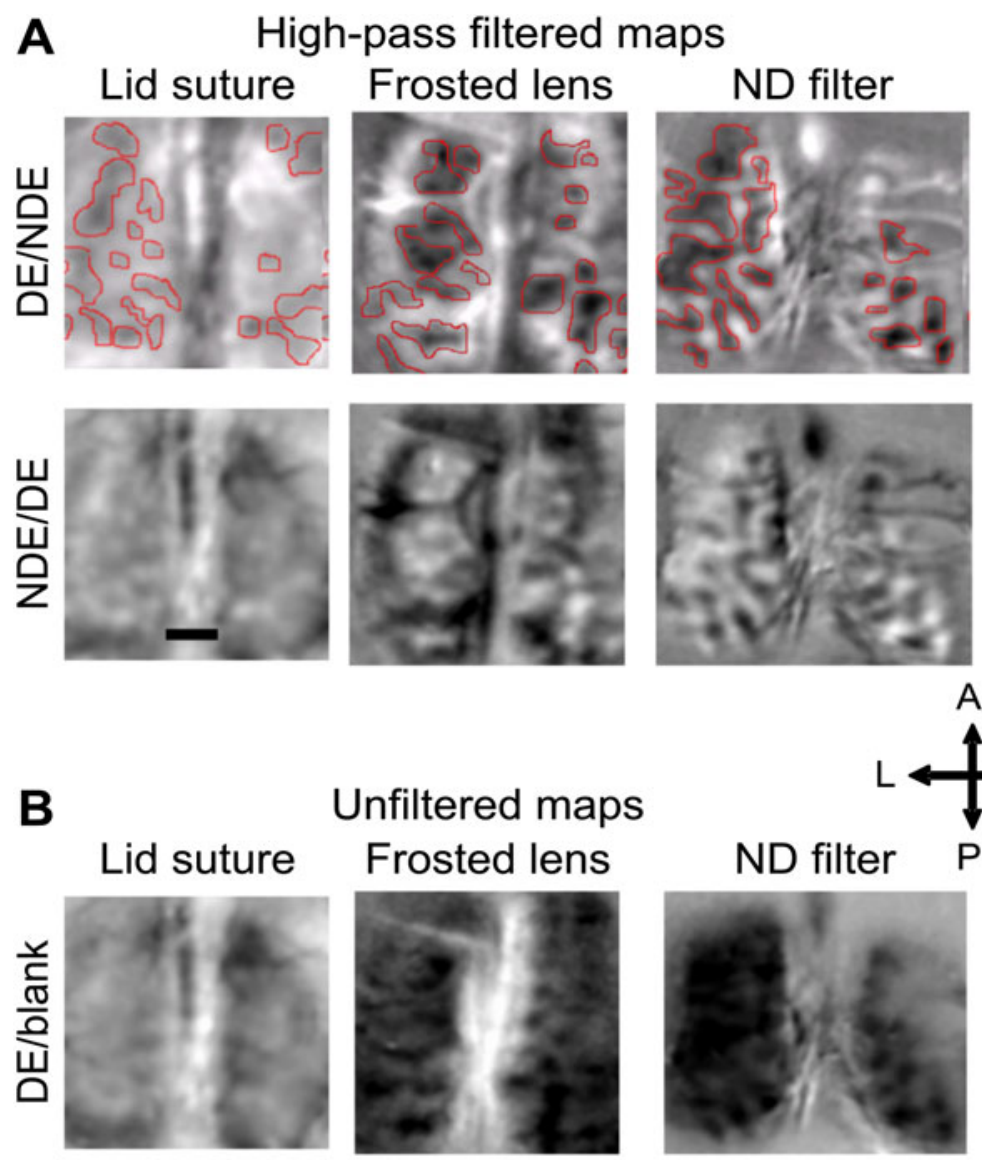

Unfiltered maps
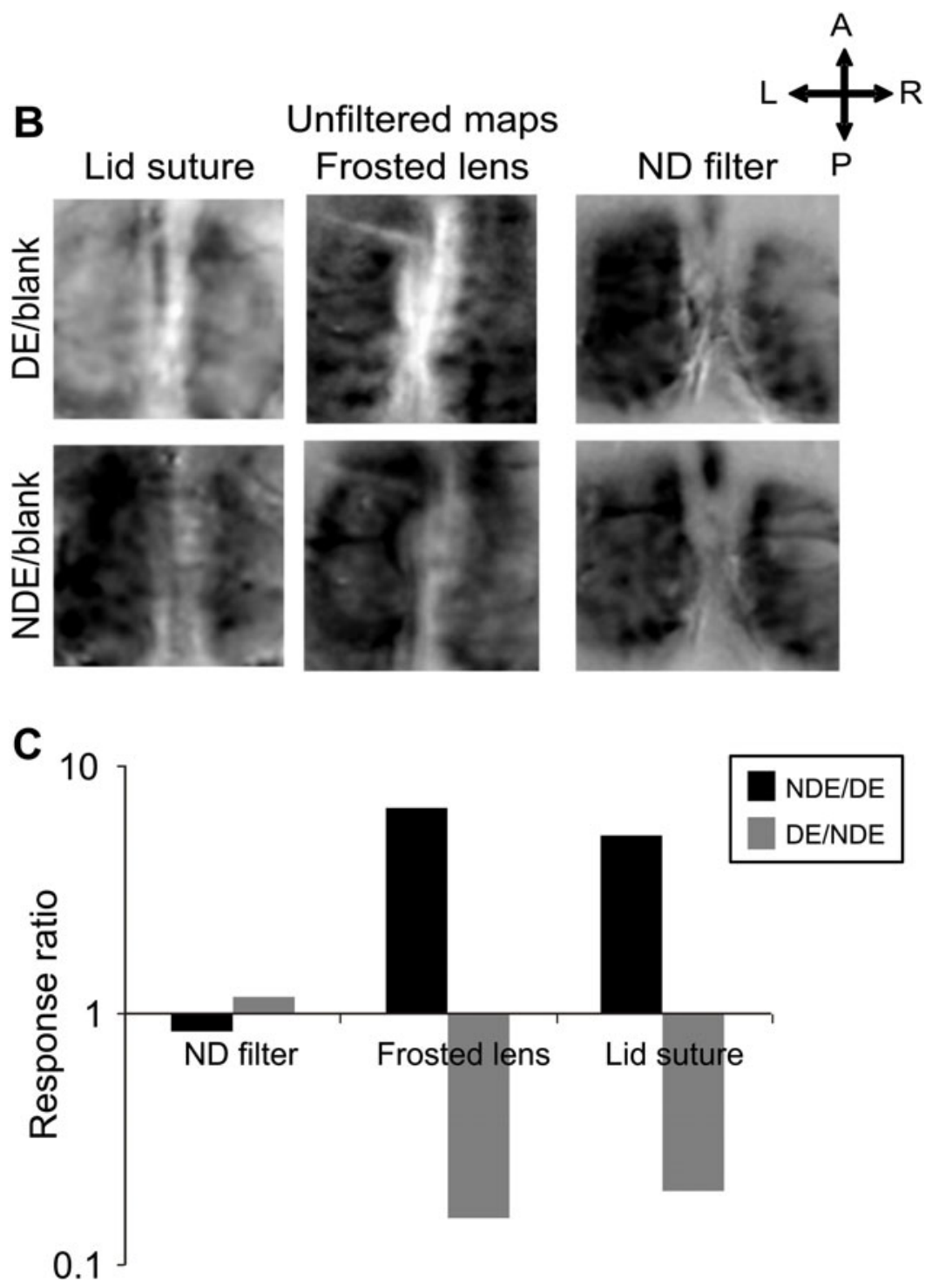

Fig. 2. Representative OD maps obtained for various MD regimens. (A) Differential high-pass filtered OD maps of subjects that underwent different forms of MD. In the row labeled DE/NDE, dark areas correspond to cortical domains activated by the DE; these are highlighted with red outlines. In the row labeled NDE/DE, dark areas correspond to cortical domains activated by the NDE. Scale bar $1 \mathrm{~mm}$. (B) Unfiltered activity maps show DE and NDE responses divided by the response to a blank screen. (C) Average ratio of response strengths through the two eyes was calculated for different MD conditions. Only MD by lid suture and MD with frosted lens caused significant differences between DE and NDE responses, that is, a response ratio significantly different from 0 . 

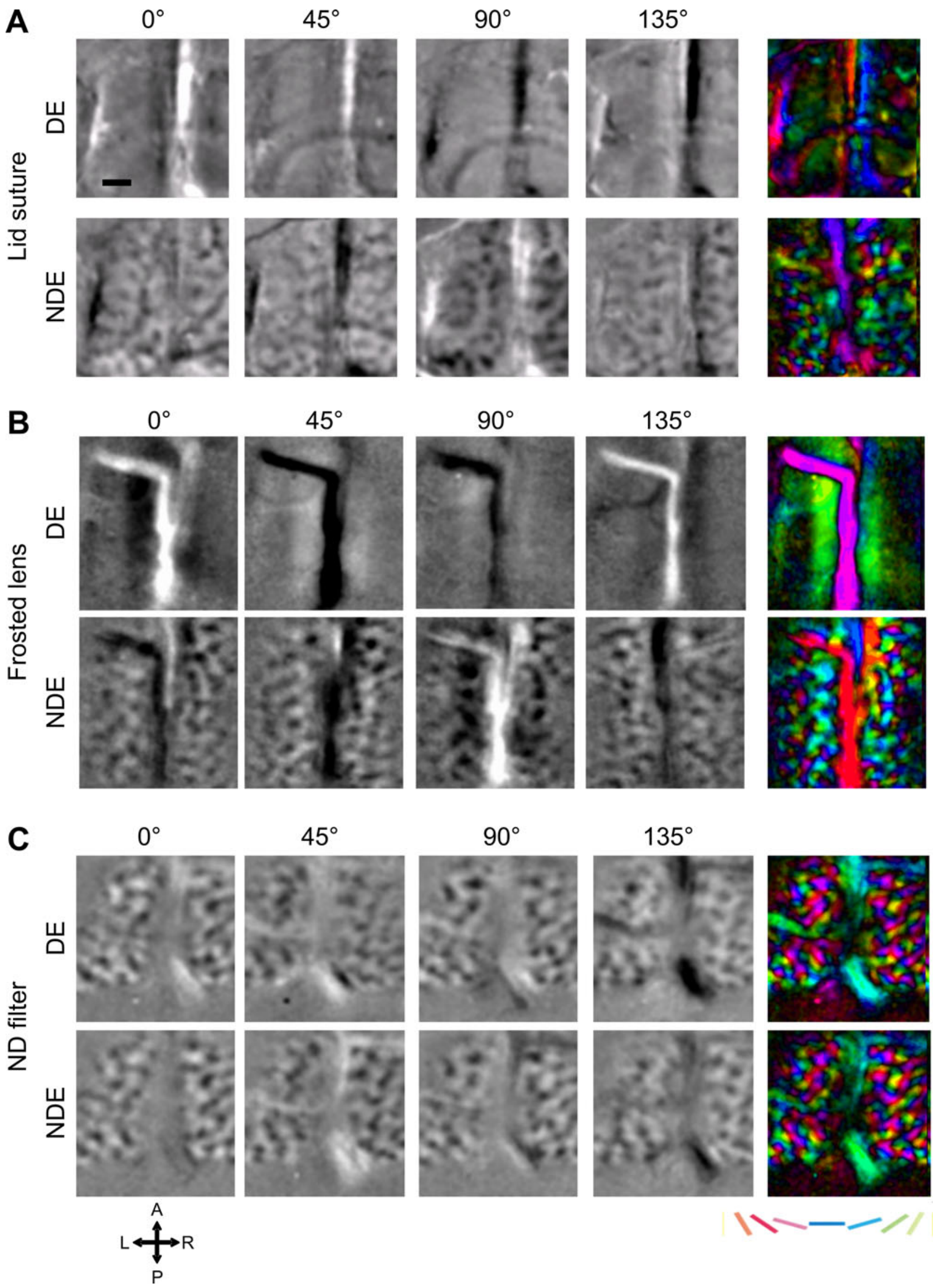
Animals were encouraged to play by providing toys such as balls to keep them awake and active during the period of visual exposure.

\section{Surgery and optical imaging}

Anesthesia was induced with an intramuscular injection of ketamine $(20-40 \mathrm{mg} / \mathrm{kg}$ ) and xylazine $(2-4 \mathrm{mg} / \mathrm{kg})$. Atropine $(0.2 \mathrm{mg} / \mathrm{kg})$ was injected intramuscularly to reduce mucous secretion. Dexamethasone was injected subcutaneously to prevent cortical edema. Animals were intubated and ventilated artificially with a mixture of $\mathrm{N}_{2} \mathrm{O}: \mathrm{O}_{2}(60: 40)$ and isoflurane (1.5-2\%). Electrocardiogram (ECG), electroencephalogram (EEG), end-tidal $\mathrm{CO}_{2}$, and rectal temperature were monitored throughout the experiment. Optical imaging of primary visual cortex was performed as described previously (Bonhoeffer \& Grinvald, 1996; Schwarzkopf et al., 2007; Hunt et al., 2009; Jaffer et al., 2012). Images were captured using an enhanced differential imaging system (Imager 2001; Optical Imaging Inc., Rehovot, Israel), with the camera focused $500 \mu \mathrm{m}$ below the cortical surface. Visual stimuli were produced by a stimulus generator (VSG Three; Cambridge Research Systems, Rochester, United Kingdom) and consisted of high contrast sinusoidally modulated gratings of two spatial frequencies (0.1-0.6 cycles/degree) and four different orientations $(0,45,90$, and 135 degrees), drifting at $2 \mathrm{~Hz}$ (maximum luminance, $76 \mathrm{~cd} / \mathrm{m}^{2}$ ). These stimuli were presented separately to the two eyes using computercontrolled shutters in randomized sequence, interleaved with four stimuli per trial in which the screen was blank. Each stimulus was first presented stationary for $1.8 \mathrm{~s}$ and then drifting for $5.4 \mathrm{~s}$. Stimulus presentations were separated by 8 -s interstimulus intervals. In total, seven blocks of data consisting of four repeats (trials) of the complete set of 20 stimuli were recorded. Image acquisition lasted for $2.5 \mathrm{~h}$. Single condition responses to drifting stimuli were added for the two eyes and divided (a) by responses to the blank screen and (b) by the sum of responses to all four orientations ("cocktail blank") to obtain iso-orientation maps. These were then band-pass filtered and range fitted for display purposes, and orientation angle maps were obtained by vectorial addition, on a pixel-by-pixel basis, of the four iso-orientation maps (Bonhoeffer \& Grinvald, 1996).

For analysis of the relative strength of responses through the two eyes, blank-divided left and right eye response images were only low-pass filtered (smoothed), and response strength was determined on a pixel-by-pixel basis in terms of by how much the value deviated from 1 (signifying a response equal to the response to a blank screen). In addition to OD, orientation selectivity was also quantified in single condition iso-orientation maps normalized by cocktail blank division. This was achieved by calculating, for all pixels within the previously defined ROI, their absolute mean deviation from 128 on a 256-point gray scale for each of the four orientations tested.

\section{Visually evoked potentials}

After imaging data acquisition, a silver ball electrode was placed on the surface of V1 near the representation of the area centralis. The recording signal was amplified by a factor of $20 \mathrm{~K}$ and bandpass filtered at 0-100 Hz. Four recordings were made (one per hemisphere and eye). The positions of the recording electrode were always mirror symmetric for the two cortical hemispheres. Stimuli were displayed on a computer screen at a distance of $57 \mathrm{~cm}$ and consisted of high contrast sinusoidally phase-reversing square-wave horizontal gratings that varied in spatial frequency from 0.14 to 3.2 cycles/deg. A blank stimulus of the same mean luminance as the gratings was used to measure spontaneous activity. A stimulus presentation lasted $3 \mathrm{~s}$, with contrast reversing at $2 \mathrm{~Hz}$, followed by an interstimulus interval of $3 \mathrm{~s}$. Stimuli were repeated 10 times in a pseudorandom order. A ratio of VEP amplitudes through the two eyes was calculated by dividing the sum of amplitudes in response to the three lowest spatial frequencies for the DE (which yielded the strongest responses in every animal) by the same sum obtained for the NDE. After data collection was completed, the animal was euthanized with an overdose of pentobarbital.

\section{Results}

For quantitative analysis of the absolute strength of responses through the DE and the NDE in both cortical hemispheres, images were only low-pass filtered (since high-pass filtering would remove absolute response level differences between the two eyes). We found that the hemispheres contralateral and ipsilateral to the DE exhibited very similar effects (MD with lid suture, MD with frosted lens, and MD with ND filter, all Kruskal-Wallis test, $P>0.05$ ), and we therefore pooled results across hemispheres.

Qualitatively, subjects that had experienced MD with lid suture or frosted lens revealed clear deprivation effects as judged by the reduced size of OD columns corresponding to the DE. Quantitatively, subjects that had experienced MD by lid suture had reduced $\mathrm{DE} / \mathrm{NDE}$ response ratios $(0.196 \pm 0.092)$, while the NDE/DE response ratio $(\Delta R / R, 5.097 \pm 2.459)$ was increased significantly (Kruskal-Wallis test, $P<0.05$ ) compared with normally reared animals (left eye/right eye response ratio; $1.129 \pm 0.105$ and right eye/left eye response ratio; $0.886 \pm 0.079)$. Similarly, subjects that had experienced MD with frosted lens had significantly (Kruskal-Wallis test, $P<0.05$ ) reduced DE/NDE response ratio $(0.152 \pm 0.061)$, while the NDE/DE response ratio was similar to the NDE/DE response ratio obtained for MD with lid suture $(6.590 \pm 0.464)$. In contrast, animals that had experienced MD by ND filter showed distinct OD patches and responses of almost equal strength to stimulation of the DE and the NDE (DE/NDE response ratio; $1.170 \pm 0.021$ and $\mathrm{NDE} / \mathrm{DE}$ response ratio; 0.854 \pm 0.135 ) (Kruskal-Wallis test, $P>0.05$ ) (Fig. 2).

Following MD by lid suture or with a frosted lens, orientation maps showed virtually no orientation-selective responses through the DE and normal responses through the NDE (Fig. 3A and 3B, respectively). In contrast, orientation maps obtained from subjects that had undergone MD with ND filter showed the pinwheel layout typical of cat $\mathrm{V} 1$ and equally strong responses to all orientations examined (Fig. 3C). This was supported by quantitative analysis revealing a very similar strength for all orientations (Kruskal-Wallis test; $P>0.05$ ) (Fig. 4).

Fig. 3. Representative orientation maps obtained for various MD regimens. Orientation maps show single condition cocktail blank-divided iso-orientation maps for the DE and the NDE obtained from an animal with MD by lid suture (A), by frosted lens (B), and by ND filter (C), respectively (scale bar $1 \mathrm{~mm}$ ). Number above the maps indicates stimulus orientation. The rightmost column shows polar maps of DE and NDE responses in which orientation-selective domains are color-coded (see color key) and response strength is indicated by intensity (Bonhoeffer \& Grinvald, 1996). 


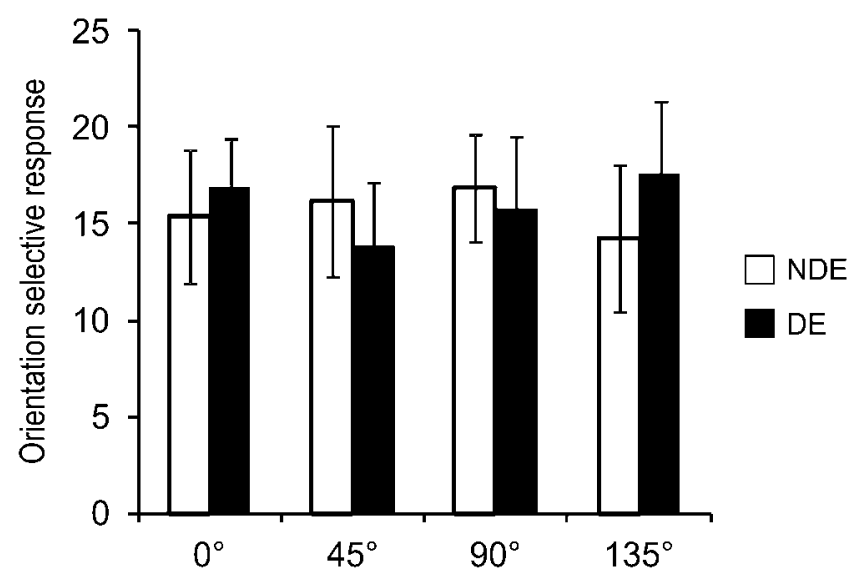

Fig. 4. Quantification of orientation-selective responses in subjects that underwent MD with ND filter. Bars show relative strength of intrinsic signals obtained for stimulation of the NDE (white bars) and the DE (black bars); the differences were not significant.

In order to assess the effects of MD on visual cortical responses to a range of spatial frequencies, VEPs were recorded. After MD with lid suture and MD with frosted lens, VEP response amplitudes were significantly reduced through the DE compared to the NDE (Kruskal-Wallis test; $P<0.05$ ) (Fig. 5A and 5B). In contrast, after MD with ND filter, VEP responses to stimulation of the DE and the NDE, respectively, did not differ significantly from each other (Kruskal-Wallis test, $P>0.05$ ) (Fig. 5C).

\section{Discussion}

Using optical imaging of intrinsic signal and VEP, we examined the physiological changes that are associated with pattern deprivation and reduced levels of illumination in the functional architecture of cat V1. In accordance with previous studies (Blakemore, 1976; Eggers \& Blakemore, 1978; Christen \& Mower, 1987; Rittenhouse et al., 2006), we show that pattern or form deprivation in one eye results in reduced responses to stimulation of that eye; in addition, we report a virtually compete loss of orientation selectivity. In contrast, attenuating retinal illumination in one eye broadly maintains cortical responses to stimulation of that eye as reported previously (Blakemore, 1976).

The present study is the first to demonstrate using optical imaging of intrinsic signals and VEPs that eliminating pattern information in one eye induces an OD shift comparable to lid suture. The reduction in VEP response amplitudes further confirms this result. We also report that near-complete depression of orientation selectivity is achieved not only by monocular lid suture but also by rearing with a frosted lens. This suggests that similar if not identical mechanisms regulate orientation selectivity and response strength after different forms of monocular pattern deprivation. Blakemore (1976) monocularly deprived animals for 37-39 h (1-2 h per day) over a period of 4 weeks, while Rittenhouse et al. (2006) deprived animals for 2 days spent in a 12-h/12-h light/dark cycle. These studies together with the present study which employed $4 \mathrm{~h}$ a day of visual exposure over 2 weeks show that various durations of daily as well as of total time spent with monocular blur result in comparable OD shifts.

The present study extends previous findings that reduced retinal illumination in one eye does not significantly affect the relative strength of responses through the two eyes; reinforcing the conclusion that pattern information drives response depression during MD as proposed previously (Blakemore, 1976; Blais et al., 1999;
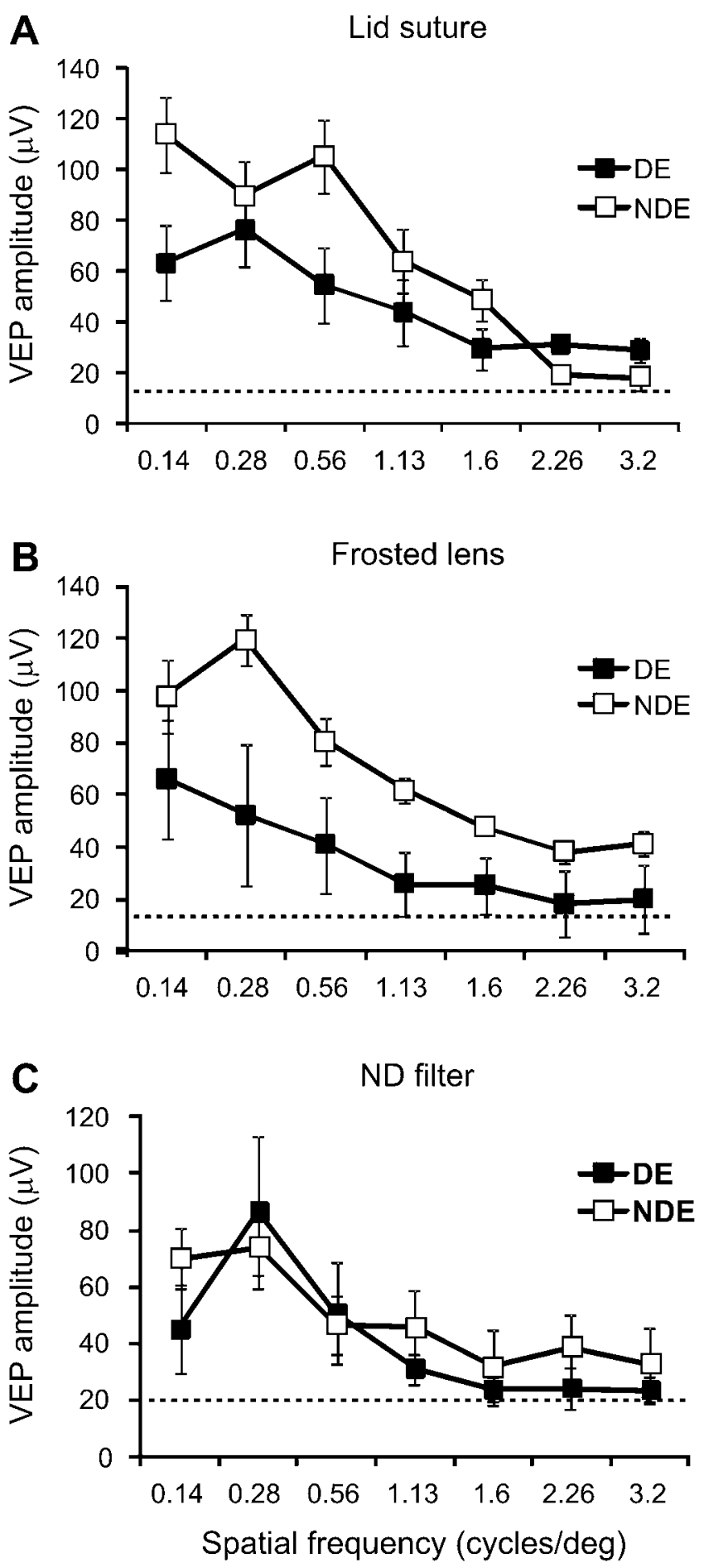

Fig. 5. Spatial frequency selectivity in cat V1 after different forms of MD. VEP signal amplitude (in $\mu \mathrm{V}$ ) for each eye, averaged across left and right hemispheres, recorded from cats with MD by lid suture (A), frosted lens (B), and ND filter (C), is plotted against spatial frequency of the stimulus. The dotted lines indicate average baseline levels of VEP amplitude in response to a blank screen. All data represent mean \pm s.E.M.

Rittenhouse et al., 2006). According to the Bienenstock-CooperMunro theory (Bienenstock et al., 1982), correlated activity arising from an open eye viewing high contrast patterns is more likely to elicit strengthening of geniculocortical synapses representing that eye, while uncorrelated activity in the DE is likely to cause 
weakening of synapses representing that eye due to lack of coincidence between pre- and postsynaptic activity (Blais et al., 1999). Notably, reduced levels of illumination in animals reared with ND filters in front of one eye cause an increase in response latency in that eye (Barlow \& Levick, 1969) and therefore asynchronous arrival of signals from the two eyes in V1, somewhat similar to strabismus. This decorrelation may contribute to a relative depletion of binocular neurons in V1 that has been observed in MD with ND filters without however affecting the balance between the two eyes (Blakemore, 1976). The key difference between the frosted lens and the ND filter is that the latter allows for normal image structure within each eye and a high degree of correlation between neighboring image points, while the former does not.

Overall, our data demonstrate that MD by lid suture and by frosted lens has similar effects on V1 OD, orientation selectivity, and VEP response amplitude due to the virtual elimination of pattern information. While the molecular basis of forms of MD other than by lid suture have not yet been investigated, we have recently shown that MD by lid suture in cats regulates the AMPA receptor GluR1 subunit as well as signaling molecules downstream of $N$-methyl-D-aspartate receptors such as PSD-95, SynGAP, and $\alpha$ CaMKII (Jaffer et al., 2012). It is likely that similar mechanisms are engaged in response to other forms of monocular pattern deprivation.

\section{Acknowledgments}

This work was supported by the U.K. Medical Research Council (grant G0500186).

\section{References}

Antonini, A. \& Stryker, M.P. (1996). Plasticity of geniculocortical afferents following brief or prolonged monocular occlusion in the cat. The Journal of Comparative Neurology 369, 64-82.

BARLOW, H.B. \& LEVICK, W.R. (1969). Changes in the maintained discharge with adaptation level in the cat retina. The Journal of Physiology, 202, 699-718.

Bienenstock, E.L., Cooper, L. \& Munro, P.W. (1982). Theory for the development of neuron selectivity: Orientation specificity and binocular interaction in visual cortex. The Journal of Neuroscience 2, 32-48.

Blakemore, C. (1976). The conditions required for the maintenance of binocularity in the kitten's visual cortex. The Journal of Physiology 261, 423-444.
BonhoEFFer, T. \& GrinVALD, A. (1996). Optical imaging based on intrinsic signals. The methodology. In: Brain Mapping: The Methods, ed. TogA, A.W. \& Mazziotta, J.C., pp. 55-97. London: Academic Press.

Blais, B.S., Shouval, H.Z. \& CoOper, L.N. (1999). The role of presynaptic activity in monocular deprivation: Comparison of homosynaptic and heterosynaptic mechanisms. Proceedings of the National Academy of Sciences of the United States of America 96, 1083-1087.

Christen, W.G. \& Mower, G.D. (1987). Effects of monocular occlusion and diffusion on visual system development in the cat. Brain Research 415, 233-241.

Crawford, M.L. \& MARC, R.E. (1976). Light transmission of cat and monkey eyelids. Vision Research 16, 323-324.

DEws, P.B. \& WIESEL, T.N. (1970). Consequences of monocular deprivation on visual behaviour in kittens. The Journal of Physiology 206, 437-455.

EgGers, H.M. \& BlaKemore, C. (1978). Physiological basis of anisometropic amblyopia. Science 201, 264-267.

FrenKel, M.Y. \& BeAR, M.F. (2004). How monocular deprivation shifts ocular dominance in visual cortex of young mice. Neuron 44, 917-923.

Hubel, D.H. \& Wiesel, T.N. (1970). The period of susceptibility to the physiological effects of unilateral eye closure in kittens. The Journal of Physiology 206, 419-436.

Hunt, J.J., Giacomantonio, C.E., Tang, H., Mortimer, D., Jaffer, S. Vorobyov, V., Ericksson, G., SengPiel, F. \& Goodhill, G.J. (2009), Natural scene statistics and the structure of orientation maps in the visual cortex. NeuroImage 47, 157-172.

Jaffer, S., Vorobyov, V., Kind, P.C. \& Sengpiel, F. (2012). Experiencedependent regulation of functional maps and synaptic protein expression in the cat visual cortex. The European Journal of Neuroscience 8, 12811294.

LoOP, M.S. \& SHERMAN, S.M. (1977). Visual discriminations during eyelid closure in the cat. Brain Research 128, 329-339.

Mrsic-Flogel, T.D., Hofer, S.B., OHKI, K., ReId, R.C., BonhoefFer, T. \& HubENER, M. (2007). Homeostatic regulation of eye-specific responses in visual cortex during ocular dominance plasticity. Neuron 54, 961-972.

Rittenhouse, C.D., Sieglar, B.A., Voelker, C.C., Shouval, H.Z., Paradiso, M.A. \& Bear, M.F. (2006). Stimulus for rapid ocular dominance plasticity in visual cortex. Journal of Neurophysiology 95, 2947-2950.

Schwarzkopf, D.S., Vorobyov, V., Mitchell, D.E. \& Sengriel, F. (2007). Brief daily binocular vision prevents monocular deprivation effects in visual cortex. The European Journal of Neuroscience. 25, 270-280.

Shatz, C.J. \& StRYKer, M.P. (1978). Ocular dominance in layer IV of the cat's visual cortex and the effects of monocular deprivation. The Journal of Physiology 281, 267-283.

Spear, P.D., Tong, L. \& Langsetmo, A. (1978). Striate cortex neurons of binocularly deprived kittens respond to visual stimuli through the closed eyelids. Brain Research 155, 141-146.

WIESEL, T.N. \& HubEL, D.H. (1963). Single-cell responses in striate cortex of kittens deprived of vision in one eye. Journal of Neurophysiology 26, 1003-1017. 